\title{
CONGENITAL DUODENAL STENOSIS DUE TO PERITONEAL BANDS.
}

\author{
BY \\ T. TWISTINGTON HIGGINS, O.B.E., F.R C.S., \\ Surgeon, Hospital for Sick Children, Great Ormond Street, \\ AND \\ DONALD PATERSON, B.A., M.B., M.R.C.P.
}

Physician to Out-Patients, Hospital for Sick Children, Great Ormond Street.

A study of the literature of this subject reveals some confusion of terms, so that it might be well at the outset to define those in common use. Stenosis, or narrowing of the duodenal tube at birth, may arise from:-

(1) Developmental error in the gut itself associated with partial or complete absence of its lumen. This is duodenal atresia.

(2) Compression or constriction of the viscus by abnormal peritoneal bands or adhesions. In this case, the gut itself possesses a normal structure, but its lumen is partially or completely obliterated by the extraneous influences.

The prognosis in these two types may be very different.

The pediatricians of this country should be pleased that by far the finest description and most extensive bibliography on this subject is by Edmund Cautley in the British Journal of Diseases of Children(1). He summarises the literature for the last hundred years and his description of the clinical picture is clearness itself. Frnst of Copenhagen(9) describes a case very like our own with operation and recovery. He, however, performed a duodeno-enterostomy. Other papers on this subject have been publisherl by Jewesbury and Max Page(5), Smellie(6), Marfan(7) and Ritter(2) and excellent observations are made on the whole subject by Cameron(3). Bosch and Schinz ${ }^{(4)}$ have described the radiographic appearances in this condition.

CASE. Baby S., Male, born October 27th, 1925. With the first feed on the second day the vomiting began. The vomit was thin, watery and green in colour. The vomiting was projectile in type, and on the third day was so forceful that it carried as far as the foot of the cot. All the vomits were green in colour. The bowels were open daily. The motions were extremely small, dark olive green in colour, and at first consisted of meconium only but later contained a few undigested curds.

The feeding consisted of half-cream Glaxo for the first week, then whey. The birth weight was 6 pounds 13 ounces. On the 9 th day after birth-seen by one of us (I. P.) - the infant was seen to be small and wasted, under nourished, with the " old man" facies so commonly seen in pyloric stenosis. The vomit was seen to be watery and bright green-undoubtedly containing bile. Subcutaneous saline was given and later a feed of bismuth carbonate in milk was administered. Dr. Bertram Shires examined the child by X-rays. The food was seen to pass easily through the pylorus and into the duodenum which ballooned out. It failed to pass further except in very small quantities ( $v$. Figs. 1 and 2, for which we are indebted to Dr. Shires). Inspection 
showed that the stomach "stood out"; no definite peristaltic waves were noted, but there was a tendency for feeble waves to start, and then fade before they had travelled far acrcss the abdomen. 'The pylorus could not be palpated although carefully felt for. In view of the clinical picture, the $X$-ray finding and the history, a diagncsis of duodenai obstruction was made, and the following day the infant was operated on.

The baby, having been anæsthetised with $\mathrm{N}_{2} \mathrm{O}$ and oxygen, the abdomen was opened by a right..paramedian incision over the gastro-ducdenal region. The state of affairs then discovered will be appreciated by referring to the drawing (Fig. 3). The stomach and first part of the duodenum were enormously distended. The pylorus could only be localised by the pyloric vein, the stomach and duodenum forming one continuous tube. The pylcric sphincter could not be detected clearly even by palpation. The cause of the duodenal obstruction was found to be the peritoneal bands, which are represented with reascnable accuracy in the drawing. Beyond the site of the duodeno-jejunal junction the gut was collapsed, except for an occasional partially expanded coil. The tethering bands were cautiously snipped thröugh with scissors and it soon became manifest that this procedure had completely relieved the obstruction. The distal coils rapidly kallocned out to a nornal size.

- Careful examination of the duodenum itself showed no sign of a genuine atresia, although at the spot where the first hand began the gut dill appear a little narrowed. In view of the fact that the obstruction had been manifestly relieved, the narrowing was not considered of serious account, and the abdomen was closed in the usual way.

The feeding after the operation was much the same as in a case of congenital pyloric stenosis. Hourly feeds of one drachm of Ideal milk mixture were given This was made up of Ideal milk one part, water four parts, demerara sugar one drachm to each four ounces of the mixture. The feed was gradually increased and the interval lengthened until on the fourth day after the operation the normal feed for the infant was reached. The bowels began to act naturally 48 hours after the operation. No vomiting occurred and the child made a rapid and apparently complate recovery. Some months later the infant appeared quite normal.

.The interest. of these cases lies in the differentiation of them from cases of congenital pyloric stenosis. In them the vomiting, which is projectile, is always definitely bile stained, and inviriably begins at birth. Peristalsis is not so well marked and no tumor can be felt. Early diagnosis and surgical treatment are necessary for recovery.

Radiography is a valuable diagnostic asset. If none of the bismuth succeeds in reaching the jejunum we may presume the presence of a complete atresia. In such a case a short circuiting operation will be necessary and the surgeon may find his efforts foiled by an atresia so extensive that no distal loop of bowel can be found with which to make the anastomosis.

Where the bismuth is observed to pass the block, the rapidity and extent to which this occurs should be carefully noted. It may be that the delay is so comparatively slight that operation is not called for, but where the clinical symptoms are at all urgent this is unlikely.

Partial obstruction, while it can occur in true atresia, is more likely to be due to adventitious bands constricting the gut. And such bands, as in this case, may be quite amenable to a comparatively simple surgical procedure. One of us (T. T. H.) has; however, recorded a case(16) where the matting: was so extensive that any attempt to free the gut would have been obviously unwise; in this case gastro-enterostomy was performed and proved successful.

It is clear therefore that surgery offers considerable hope in these cases and will practically alwars be recessary for recovery. 


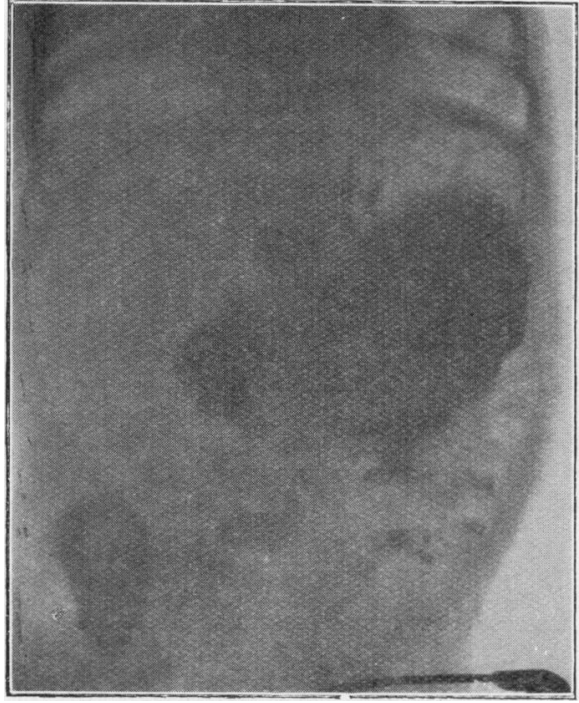

Fig. 1.

Immediately after meal.

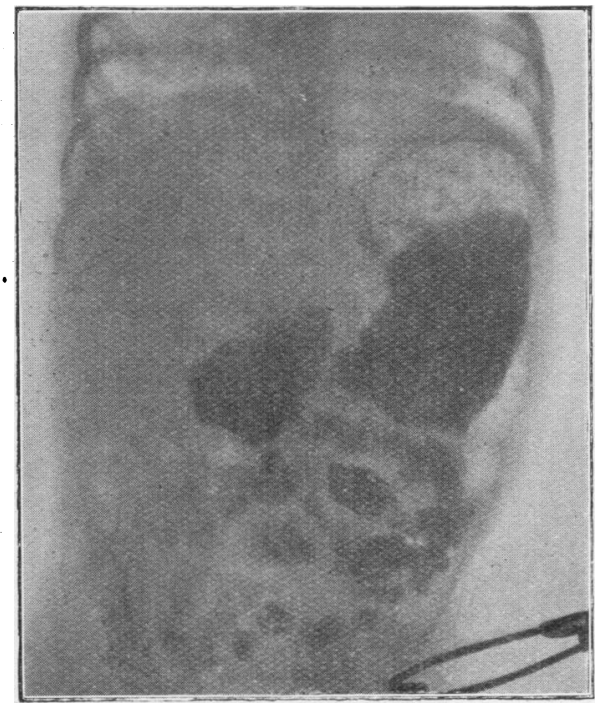

Firg.'.2.

One hour after meal.

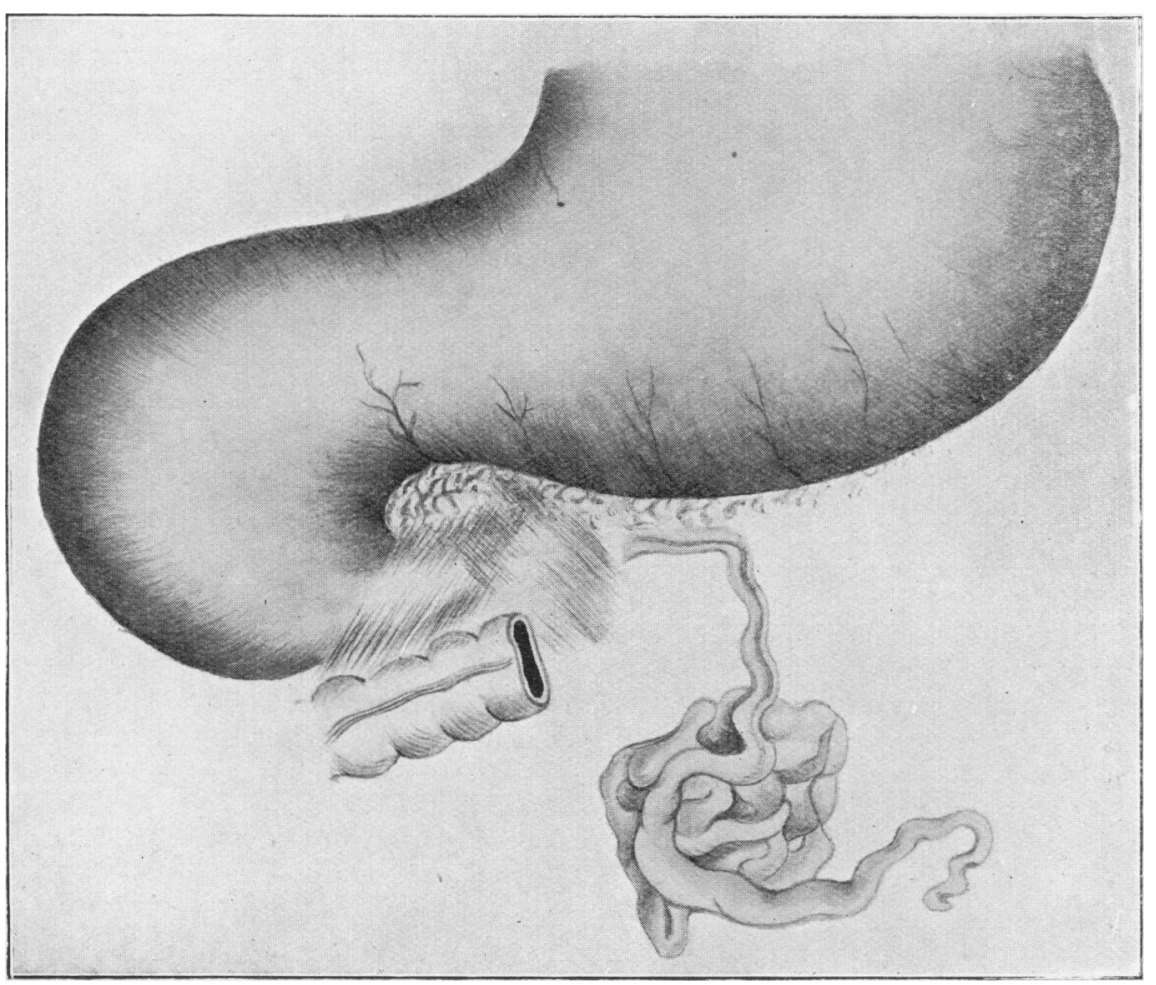

Fig. 3. 


\section{BIBLIOGRAPHY.}

1. Cautley, E., Brit. Jour. Dis. Ch., 1919, XVI, 65.

2. Ritter, C., Jahrb. f. Kinderh., Berlin, $192 \overline{0}, \mathrm{XCT}, 369$.

3. Cameron, H. C., Brit. Med. Jour., 1925, I, 765, 815, 872.

4. Bosch, E., and Schinz, H. R., Deutsche. Ztschr. f. chir., Leipsig, 1920, CI., 284.

5. Jewesbury, R. C., and Page, Max, Proc. Roy. Soc. Med., 1923, XVI (Sec. Dis. Ch.), 50.

6. Smellie, J. M., Brit. J. Dis. Ch., 1924, XXI, 192.

7. Marfan, A., Turquety, and Debray, J., Nourrisson, Paris, 1924, XII, 101.

8. Neff, F. C., and Haden, R. L., Amer. Jour. Dis. Ch., Chicago, 1925, XXX, 82

9. Ernst, N. P., Brit. Med. Jour., 1916, I, 644.

10. Roe, W. R., and Shaw, E. H., Lancet, 1911, II, 947.

11. Koos, A. V., Jahrb. f. Kinderhm., Berlin, $1 \overline{920}$, XCV, 240.

12. Salzmann, M., Monatschr. f. Kinderh., Leipsig, 1921, XIX, 468.

13. Jewesbury, R. C., Proc. Roy. Soc. Med., 1922, XVI (Sec. Dis. Ch.), 10.

14. Denzer, B. S., Am. J. Dis. Ch., Chicago, 1922, XXIV, 534.

15. Stransky, E., Med. Klinik, Leipsig, 1922, XVIII, 1433.

16. Higgins, T. Twistington, Brit. Jour. Surgery, Bristol, 1924, XI, $3 \overline{8} \overline{2}$. 